\title{
PTCH1 Germline Mutations and the Basaloid Follicular Hamartoma Values in the Tumor Spectrum of Basal Cell Carcinoma Syndrome (NBCCS)
}

\author{
GIOVANNI PONTI ${ }^{1}$, MARCO MANFREDINI $^{2}$, LORENZA PASTORINO $^{3,4}$, \\ MONIA MACCAFERRI ${ }^{1}$, ALDO TOMASI $^{5}$ and GIOVANNI PELLACANI ${ }^{2}$ \\ ${ }^{1}$ Clinical Pathology Unit, Surgical, Medical and Dental Department of Morphological Sciences related to Transplant, \\ Oncology and Regenerative Medicine, University of Modena and Reggio Emilia, Modena, Italy; \\ ${ }^{2}$ Dermatology Unit, Surgical, Medical and Dental Department of Morphological Sciences related to Transplant, \\ Oncology and Regenerative Medicine, University of Modena and Reggio Emilia, Modena, Italy; \\ ${ }^{3}$ Department of Internal Medicine, Medical Specialties and Surgical Science and Integrated Diagnostics, \\ University of Genoa, Genoa, Italy; \\ ${ }^{4}$ Genetics of Rare Cancers, IRCCS AOU San Martino-IST Istituto Nazionale per la Ricerca sul Cancro, Genoa, Italy; \\ ${ }^{5}$ Department of Diagnostic and Clinical Medicine and Public Health, \\ University of Modena and Reggio Emilia, Modena, Italy
}

\begin{abstract}
Background/Aim: Nevoid basal cell carcinoma syndrome (NBCCS) is an autosomal dominantly inherited disorder characterized by multiple basal cell carcinomas $(B C C)$, odontogenic tumors and various skeletal anomalies. Basaloid follicular hamartomas (BFHs) constitute rare neoplasms that can be detected in sporadic and familial settings as in the Basaloid Follicular Hamartoma Syndrome (BFHS). Although BFHS shares clinical, histopathological and genetic overlapping with the NBCCS, they are still considered two distinctive entities. The aim of our singleinstitution study was the analysis of a cohort of PTCH1mutated patients in order to define clinical and biomolecular relationship between NBCCS and BFHs. Materials and Methods: In our study we evaluated PTCH1 gene-carrier probands affected by NBCCS to detect the incidence of BFHs and their correlation with this rare syndrome. Results: Among probands we recognized 4 patients with BFHs. We found 15 germline PTCH1 mutations, uniformly distributed across the
\end{abstract}

Correspondence to: Dr. Giovanni Ponti, Department of Surgical, Medical, Dental and Morphological Sciences with Interest Transplant, Oncological and Regenerative Medicine, Clinical Pathology Unit, University of Modena and Reggio Emilia, Modena, Via del Pozzo $\mathrm{n}^{\circ}$ 71, 41124, Italy. Tel: +39 0594224264, Fax +39 0594224271, e-mail: giovanni.ponti@unimore.it

Key Words: Nevoid basal cell carcinoma syndrome, basaloid follicular hamartoma, PTCH1, basaloid follicular hamartoma syndrome, basal cell carcinoma, hereditary cancer.
PTCH1 gene. Six of them had familial history of NBCCS, two of them were novel and have not been described previously. Conclusion: NBCCS and BFHS may be the same genetic entity and not two distinctive syndromes. The inclusion of $\mathrm{BFH}$ in the NBCCS cutaneous tumor spectrum might be useful for the recognition of misdiagnosed NBCCS cases that could benefit from tailored surveillance strategies.

Nevoid basal cell carcinoma syndrome (NBCCS) (OMIM \#109400), also known as Gorlin syndrome (GS) is an autosomal-dominant inherited disorder characterized by predisposition to the development of basal cell carcinomas (BCCs) along with various dental, osseous, ophthalmic, and neurological abnormalities $(1,2)$. A wide variety of benign or malignant tumors can be found in association with these syndromes, such as ovarian fibroma, medulloblastoma, rhabdomyosarcoma, cardiac fibroma and ameloblastoma (1). NBCCS is correlated with $P T C H 1$ germline mutations, but in this hereditary setting the genotype- phenotype correlation is not always present. In fact, gene carriers and family members that present the same PTCHI germline mutation have a variable skin tumor spectrum phenotype $(3,4)$ including pigmented BCCs, infundibulocystic BCC, cystic BCCs, Pinkus fibroepithelioma (5), and other benign neoplasms as ovarian fibroma, medulloblastoma, rhabdomyosarcomas, cardiac fibromas and ameloblastoma (1). Basaloid follicular hamartoma $(\mathrm{BFH})$ is a rare benign skin appendage tumor that presents hair follicle differentiation and may be a familial, congenital or acquired condition (6). This tumor appears as small skin-coloured or yellowish or brownish papule and is 
often confused and diagnosed as BCC or trichoepithelioma. Five clinical forms of BFH have been noted: (1) solitary or multiple papules, (2) a localized plaque with alopecia, (3) a localized linear or unilateral papule or plaque, (4) a generalized dominantly inherited familial type without associated disorders, or (5) generalized papules associated with alopecia and myasthenia gravis $(6,7) . \mathrm{BFH}$ is histologically characterized by malformed and distorted hair follicles composed of cords and strands of basaloid cells (8). Multiple basaloid follicular hamartomas may be localized or generalized and generally involve the face, scalp, neck and rarely the trunk. It is a rare type of lesion whose finding is not considered by many authors as a significant criterium for the diagnosis of NBCCS. They can be revealed in generalized basaloid follicular hamartoma syndrome (GBFHS), segmentally arranged basaloid follicular hamartoma syndrome, in association with other genodermatoses or autoimmune disorders (myasthenia gravis, alopecia universalis, cystic fibrosis and systemic lupus erythematosus) $(7,9,10)$.

NBCCS and BFHS share some common distinctive signs, in particular palmo-plantar pits and milia are signs that are present in both syndromes (11). In addition to that, some of the features of NBCCS often recur in patients affected by BFHS and vice versa, for example: two siblings of African ethnicity affected by BFHS presented broadened nasal root and frontal bossing (12) and many patients with NBCCS can be affected by BFHSassociated milia (7) and/or alopecia (10). The differential diagnosis among BCC and BFH or other benign and malignant skin tumors could be improved by the use of dermoscopy and other modern imaging techniques, in detail by reflectance confocal microscopy (13). The aim of our study was the analysis of the clinico-pathologic and biomolecular relationship between NBCCS and BFHS; the specific aim was the evaluation of the role of BFHs in the NBCCS cutaneous tumor spectrum and its association with $\mathrm{PTCH}$ gene mutation status.

\section{Patients and Methods}

NBCCS patients were evaluated in the Departments of Pathology and Dermatology of the University of Modena and Reggio Emilia, Italy. NBCCS diagnostic criteria were those updated by Kimonis et $a l$. in 1997 (14) and detailed by Evans and Farndon in 2002 (15). Diagnosis of NBCCS was established when 2 major or 1 major and 2 minor criteria were present. A set of dermoscopic pictures and reflectance confocal microscopy images of BCCs and BFHs were acquired and collected. Blood samples were drawn from patients following provision of informed consent. Paraffin embedded tumor tissues were collected and evaluated through immunohistochemical and biomolecular analysis. This study was approved by the Ethics Committee of the University hospital of Modena.

PCR amplification and sequencing. For testing of PTCH1, genomic DNA was extracted with the NUCLEON BACC3 kit (Amersham Pharmacia Biotech, Milan, Italy) and coding regions including exon-intron boundaries and splice-site junctions were sequenced in forward and reverse orientations by using the ABI BigDye
Terminator Ready Reaction Mix (Applied Biosystems, Foster City, CA, USA) and analyzed on an ABI 3130XL Genetic Analyzer according to the manufacturer's protocol. A detailed list of the primers and PCR conditions are available upon request. The PTCH1 cDNA sequence from GenBank (Accession number NM_000264.3) was used as a reference sequence.

\section{Results}

We found 15 PTCH1 germline mutations in 16 probands that belonged to families affected by NBCCS. Four of these probands $(20 \%)$ presented multiple BFHs, with early age of onset. In this cohort the BFHs were predominantly located on the head and neck area, mainly on the cheek and forehead region. In one family we found a proband with generalized BFHs showing multiple lesions that were uniformly distributed on his body surface. In the other two probands we detected localized BFHs (Figure 1a-e). In all 4 families we found the full NBCCS diagnostic criteria (Table I), while we didn't find the known potentially BFHS associated diseases, such as: myasthenia gravis, alopecia universalis, cystic fibrosis and systemic lupus erythematosus. In detail, among BFHs probands and in their first and second-degree relatives, very few BCCs were diagnosed. In spite of a relatively small number of BCCs detected in these three NBCCS probands affected by multiple BFHs, many of the other criteria for NBCCS diagnosis were found: distinctive skeletal signs, characteristic macrocephaly, asymmetric facies and positive history of odontogenic keratocystic tumors were all found in the three patients. In particular, the few BCCs of two patients represented the cause of an important NBCCS diagnostic delay. Regarding the other 12 NBCCS probands with a typical skin tumor spectrum characterized by multiple and recurrent BCCs, its number varied from five to several hundreds and ranged in size from 1 to $45 \mathrm{~mm}$ in diameter (Table I). Three patients excised Pinkus fibroepitheliomas, three had also palmo-plantar pits, one presented multiple caffé- au-lait macules.

Histopathological examination of BFHs revealed malformed and distorted hair follicles composed of cords and strands of basaloid cells (Figure 1f, g). Generally, the surrounding stroma was few or absent and the mitotic activity was rare or absent. Usually, the tumor cells were bland without nuclear pleomorphism and mucinous ground substance was absent or subtle. Peripheral palisading and clefts within the fibrous stroma can be observed. Differently, histopathological examination of BCCs showed cords and island of basaloid cells embedded in a mucinous matrix and often surrounded by fibroblast and lymphocytes. Peripheral palisading and clefts within the fibrous stroma were usually observed. Moreover, increased mitoses and single cells necrosis were frequently identified. In BCCs, the tumor could destroy hair follicles and infiltrate the interfollicular dermis, while in BFHs, the neoplastic process was seen only 

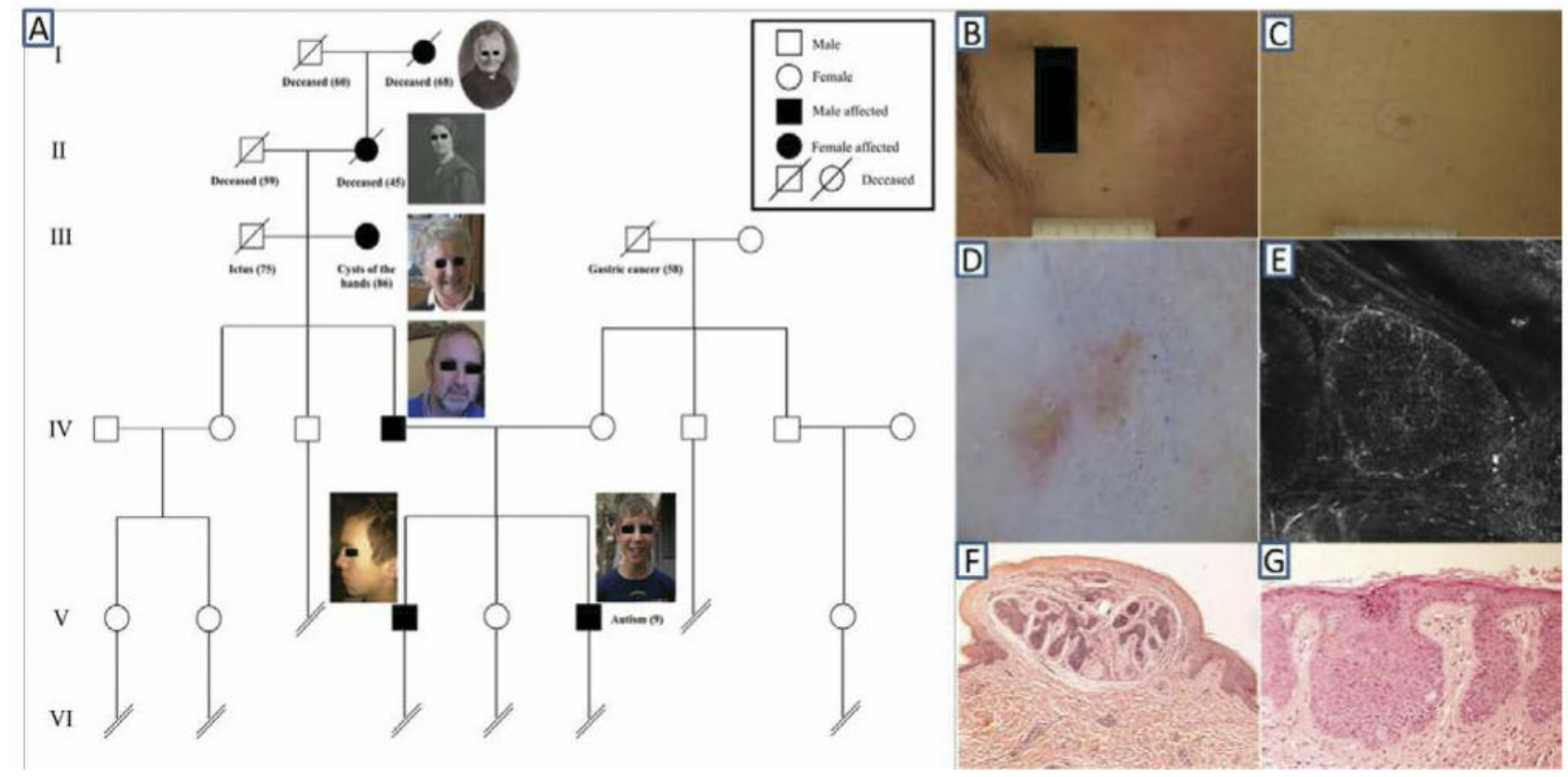

Figure 1. Clinical features of BFH probands and in their first and second-degree relatives (a). Clinical presentation of facial BFHs (b, $c)$; Dermoscopic features of BFH $(d)$; RCM image of BFH showing basaloid nests with reduced clefting phenomena (e); Histologic features $(f$, g). Basaloid follicular hamartoma shows strands and cords of small, basaloid cells emanating from the infundibular portion of the hair follicle. The tumor stroma was scant and mildly fibrocellular (hematoxylin-eosin).

where normal hair follicles were present. Moreover, in BFH interfollicular dermis was never interested.

In our series, BFHs consisted of pink papules, slightly infiltrated, firm and with a translucent surface, predominantly localized on the face, but also distributed to the trunk, arms and hands. Dermoscopic images of two small lesions showed the presence of short fine telangiectasia in both of them (Figure 1d) and gray- brown globules in one of them. Reflectance confocal microscopy (RCM) showed epithelial cords in the upper dermis and basaloid-like islands of ovoid shape in both lesions (Figure 1e). Clefting appeared to be reduced but further analysis on larger populations need to be performed. Histologically all the lesions showed similar features, presenting cords of basaloid cells in the upper dermis arranged mainly in a radial and anastomosing fashion. The cells had regular nuclei, and mitotic activity was absent. Stroma was scant and minimal clefting between the tumor and the stroma was observed. The results of the molecular analysis of consenting Italian patients with histologically confirmed NBCCS are shown in Table I. We found 15 germline $P T C H 1$ mutations, which appeared to be uniformly distributed across the PTCHI gene. Six of them belonged to patients with a family history of NBCCS. To our knowledge, two of them (14.3\%) were novel and had not been previously described (Probands 15 and 16 of Table I).
In detail, PTCH1 mutations associated to BFHs don't show any correlation with a distinctive clinical phenotype, being distributed along the whole gene sequence and not being associated to any mutation hotspot (Table I).

\section{Discussion}

The evaluation of the clinico-pathological and biomolecular relationship between NBCCS and BFHS may support the overlap of the clinical and genetic features of the affected individuals. The PTCH1 mutations seem to be involved in the pathogenesis of multiple and recurrent BFHs as well as multiple and recurrent BCCs in the NBCCS setting. We consider that the recognition of BFHs in the cutaneous tumor spectrum of three NBCCS probands gene-carriers of germline PTCH1 is a further step towards the inclusion of BFHs into NBCCS-diagnostic criteria.

The common molecular pathogenesis of NBCCS and BFHS is demonstrated by the analysis of the PTCHI sequence, whose germline mutations give rise to both these syndromes (Table I). Even though they share many genetic and clinical features, they are not considered as the same entity by many author (16). Although, multiple BFHs have been described in NBCCS patients $(17,18)$ and the skin hamartomas of BFHS have an increased risk of progression 
Table I. Patients, PTCH1 mutations and clinical features.

\begin{tabular}{|c|c|c|c|c|c|c|c|}
\hline $\mathrm{Pt}$ & Gender & Age & $\begin{array}{l}\text { Gene } \\
\text { mutation }\end{array}$ & $\begin{array}{l}\text { Mutation } \\
\text { type }\end{array}$ & $\begin{array}{l}\text { Cutaneous } \\
\text { fenotype }\end{array}$ & $\begin{array}{l}\text { Other phenotypes } \\
\text { associated }\end{array}$ & $\begin{array}{c}\text { Relatives } \\
\text { affected }\end{array}$ \\
\hline 1 & M & 41 & $\begin{array}{l}\text { c. } 1987 \mathrm{C}>\mathrm{T} \\
{[\mathrm{p} . \mathrm{Q} 663 \mathrm{X}]}\end{array}$ & Nonsense & $\mathrm{BCC}$ & KCOT, Sternum cyst & No \\
\hline 2 & $\mathrm{~F}$ & 22 & c.2629delT & Frameshift & BFHs, BCC & KCOT, Falx cerebri calcification & \\
\hline 3 & $\mathrm{~F}$ & 42 & c. $585-1 \mathrm{G}>\mathrm{A}$ & Splicing & BCC & KCOT & Father sister, \\
\hline 4 & M & 55 & $\begin{array}{l}\text { c. } 2062 \mathrm{C}>\mathrm{T} \\
{[\mathrm{p} . \mathrm{Q} 688 \mathrm{X}]}\end{array}$ & Nonsense & $\mathrm{BCC}$ & KCOT, Bifid rib & Daughter \\
\hline 5 & M & 67 & $\begin{array}{l}\text { c. } 1237 \mathrm{C}>\mathrm{T} \\
{[\mathrm{p} . \mathrm{Q} 413 \mathrm{X}]}\end{array}$ & Nonsense & $\begin{array}{l}\text { BFHs, BCC, Palmo- } \\
\text { plantar pits }\end{array}$ & $\begin{array}{c}\text { KCOT, Falx cerebri } \\
\text { calcification, Macrocephaly }\end{array}$ & $? ? ?$ \\
\hline 6 & M & 48 & c. $654+2 \mathrm{~T}>\mathrm{A}$ & Splicing & $\mathrm{BCC}$ & $\begin{array}{l}\text { KCOT, Falx cerebri calcification, } \\
\text { macrocephaly Hydrocefalus, } \\
\text { Bifid rib, Polydactylism left foot }\end{array}$ & Father \\
\hline 7 & $\mathrm{~F}$ & 63 & c.931ins A & Frameshift & $\mathrm{BCC}$ & $\begin{array}{l}\text { KCOT, Polydactily, Bifid rib, } \\
\text { Ovarian fibromas; Myopia, AML }\end{array}$ & Father \\
\hline 8 & $\mathrm{~F}$ & 45 & $\begin{array}{l}\text { c.3277G }>A \\
\text { [p.G1093R] }\end{array}$ & Missense & $\begin{array}{l}\text { BCC, Palmo- } \\
\text { plantar pits }\end{array}$ & KCOT & Father \\
\hline 9 & M & 63 & c.1041delAA & Frameshift & $\mathrm{BCC}$ & KCOT, Bifid rib & \\
\hline 10 & M & 42 & $\begin{array}{l}\text { c. } 2186 \mathrm{~A}>\mathrm{T} \\
{[\mathrm{p} . \mathrm{K} 729 \mathrm{M}]}\end{array}$ & Missense & $\mathrm{BCC}$ & KCOT, AML, Skeletal anomalies & Son \\
\hline 11 & M & 70 & c.1253delA & Frameshift & BCC, Palmo-plantar pits & KCOT, Macrocephaly & First degree relative. \\
\hline 12 & M & 18 & c.1134insG & Frameshift & BFHs, BCC & $\begin{array}{l}\text { KCOT, Falx cerebri calcification, } \\
\text { Macrocephaly, Bifid rib }\end{array}$ & \\
\hline 13 & M & 60 & c. $1348-2 \mathrm{~A}>\mathrm{G}$ & Splicing & $\begin{array}{l}\text { BCC, Epidermoid cyst, } \\
\text { Cafe au lait spots }\end{array}$ & $\mathrm{KCOT}$ & Father Brother \\
\hline 14 & M & 84 & - & & BFHs generalized type & KCOT & \\
\hline 15 & $\mathrm{~F}$ & 39 & c.2276delG & Frameshift & $\mathrm{BCC}$ & Epidermoid cysts, Macrocephaly & \\
\hline 16 & $\mathrm{~F}$ & 24 & $\begin{array}{l}\text { c. } 2532 \mathrm{G}>\mathrm{C} \\
\text { [p.W844C] }\end{array}$ & Missense & $\mathrm{BCC}$ & KCOT, Macrocephaly & \\
\hline
\end{tabular}

BCC: Basal cell carcinoma; BFH: basaloid follicular hamartoma; KCOT: keratocystic odontogenic tumor.

to BCCs, these two syndromes are still not considered as the same genetic entity as suggested by Ackerman AB (19). In 2009, Ackerman wrote a letter in which he focused on the differentiation between BFHs and infundibulocystic BCC, stating that "in his view, generalized basaloid follicular hamartoma syndrome (GBFHS) in actuality is the nevoid basal cell carcinoma syndrome" (19).

It is plausible to hypothesize that the variability of the skin neoplastic spectrum in NBCCS does include also the BFHs, as a characteristic feature of the syndrome. The analysis of our cohort of PTCHI gene carrier probands support the theory that BFHs are uncommon tumors which are associated to less severe NBCCS cutaneous phenotype. In detail, the number of BCC and other cutaneous manifestations in those $\mathrm{PTCH}$ l gene carriers with diagnosis of BFHS were fewer and less severe than in the classic NBCCS phenotype. The wide phenotypic changes are often associated to the presence of complex and variable genotype alterations, which affect $P T C H 1$ through many different mutations. Concerning the differential diagnosis, BFH may be misdiagnosed into many other skin tumor, such as BCC, trichilemmoma and trichoepithelioma. In detail the differentiation between BFH and BCC is complex both for the clinician and the pathologist. Modern non-invasive imaging approaches, such as RCM, can be a precious aid to the clinician for the diagnosis of these lesions and for the recognition of patients that should be treated with the surgical excision of the tumors instead of other ablative treatments such as cryotherapy, $\mathrm{CO}_{2}$ laser treatment or Imiquimod based treatment regimens. In addition, the diagnosis of the tumors at an early stage of development, when their diameter is below $5 \mathrm{~mm}$, helps to minimize surgical excisions and unwanted aesthetic side effects. Concerning pathologic diagnosis, the immunohistochemistry may help the recognition of the BCC through the evaluation of positive $\mathrm{Ki}-67$ and Bcl-2 staining, whereas CD-34 staining is absent in BCCs and positive in the stromal cells next to the tumor cells in BFH (8).

Genetic studies have linked BFHs affected patients to PTCH1 gene mutations, even though the phenotypic expression of NBCCS patients with BFH lesions have a less severe skin phenotype in respect to the classic NBCCS 
phenotype (20). Mutations in PTCHI have been recognized in different skin neoplasms including sporadic BCCs, trichoblastoma, trichoepithelioma, and dentigerous cysts (21). The analysis of genotype-phenotype correlations of our NBCCS series showed lack of genotype-phenotype correlation (22-25). Regarding the Brazilian NBCCS family (case 15), the frameshift mutation identified is common and not specifically linked to other $\mathrm{PTCH} 1$ germline mutations recognized in the Brazilian NBCCS series reported by da Silva Pierro VS (26). However, the phenotypic characterization of our Brazilian kindred was in accordance with the clinical behavior reported by da Silva Pierro VS (26), which describes NBCCS patients with less aggressive cutaneous tumor spectrum. This is probably explained by the high concentration of melanin, which has a protective role against UV radiations and has been associated with a decreased chance of developing BCC. The wide variability in the NBCCS tumor spectrum could be addressed to the second mutation in the $S U F U$ gene or other genes related to PTCH1 which can act as modifier genes influencing the neoplastic phenotype.

The role of BFH diagnosis, in particular for the probands with suspected NBCCS diagnosis in which the cutaneous diagnostic criteria are not completely satisfied ( $\mathrm{BCC}<5$ after 30 y.o. or $<1$ before 30 y.o.) can be crucial for the early diagnosis of the syndrome and for their tailored clinical management. The consequences of our epidemiologic and biomolecular analysis can be summarized in the follow main point: first $\mathrm{BFH}$ is a rare entity either in the general population or in the NBCCS setting, therefore it may be useful as a clinical marker of the hereditary disease; second: the NBCCS cutaneous tumor spectrum should include a wide range of benign $(\mathrm{BFH}$, milia, palmoplantar pits, epidermoidal cysts) and malignant lesions including different types of $\mathrm{BCCs}$ (pigmented BCCs, infundibulocystic BCC, cystic $\mathrm{BCCs}$, epidermoidal cysts, fibroepithelioma of Pinkus); third: it is important to suggest the suspicion of NBCCS wherever multiple BFHs are recognized in association to other NBCCS clinical and biomolecular criteria, even in the absence of the classic cutaneous phenotype.

It is important to underline the clinical impact of a BHF, which could influence the early NBCCS diagnosis, thus constituting an important feature that should be considered for the management and genetic counseling of potentially NBCCS-affected patients. The future challenges of the biomolecular research on the pathogenesis of the syndrome will be focused on the identification of the modifier genes, or other molecular targets, implied in the development of the cutaneous phenotypic variability of the syndrome.

\section{Conflicts of Interest}

There are no conflicts of interest.

\section{Acknowledgements}

Honorarium, grant, or other form of payment were not given to anyone of the authors to produce the manuscript. All Authors made substantive intellectual contributions to the published study and each author listed on the manuscript has seen and approved the submission of the manuscript.

\section{References}

1 Kiwilsza M and Sporniak-Tutak K: Gorlin-Goltz syndrome a medical condition requiring a multidisciplinary approach. Med Sci Monit 18: RA145-153, 2012.

2 Pastorino L, Pollio A, Pellacani G, Guarneri C, Ghiorzo P, Longo C, Bruno W, Giusti F, Bassoli S, Bianchi-Scarrà G, Ruini C, Seidenari S, Tomasi A and Ponti G: Novel PTCH1 mutations in patients with keratocystic odontogenic tumors screened for nevoid basal cell carcinoma (NBCC) syndrome. PLoS One 7: e43827, 2012.

3 Ponti G, Pollio A, Mignogna MD, Pellacani G, Pastorino L, Bianchi-Scarrà G, Di Gregorio C, Magnoni C, Azzoni P, Greco M and Seidenari S: Unicystic ameloblastoma associated with the novel K729M PTCH1 mutation in a patient with nevoid basal cell carcinoma (Gorlin) syndrome. Cancer Genet 205: 177-181, 2012.

4 Ponti G, Pollio A, Pastorino L, Pellacani G, Magnoni C, Nasti S, Fortuna G, Tomasi A, Scarrà GB and Seidenari S: Patched homolog 1 gene mutation (p.G1093R) induces nevoid basal cell carcinoma syndrome and non-syndromic keratocystic odontogenic tumors: a case report. Oncol Lett 4: 241-244, 2012.

5 Go JW, Kim SH, Yi SY and Cho HK: Basal cell nevus syndrome showing several histologic types of Basal cell carcinoma. Ann Dermatol 23: S36-40, 2011.

6 Wheeler CE Jr., Carroll MA, Groben PA, Briggaman RA, Prose NS and Davis DA: Autosomal dominantly inherited generalized basaloid follicular hamartoma syndrome: report of a new disease in a North Carolina family. J Am Acad Dermatol 43: 189-206, 2000.

7 Gumaste P, Ortiz AE, Patel A, Baron J, Harris R and Barr R: Generalized basaloid follicular hamartoma syndrome: a case report and review of the literature. Am J Dermatopathol 37: e3740, 2015.

8 Mills $\mathrm{O}$ and Thomas LB: Basaloid follicular hamartoma. Arch Pathol Lab Med 134: 1215-1219, 2010.

9 Brown AC, Crounse RG and Winkelmann RK: Generalized hairfollicle hamartoma, associated with alopecia, aminoacidura, and myasthenia gravis. Arch Dermatol 99: 478-493, 1969.

10 Gorlin RJ: Nevoid basal-cell carcinoma syndrome. Medicine (Baltimore) 66: 98-113, 1987.

11 Ramos-Ceballos FI, Pashaei S, Kincannon JM, Morgan MB and Smoller BR: Bcl-2, CD34 and CD10 expression in basaloid follicular hamartoma, vellus hair hamartoma and neurofollicular hamartoma show full follicular differentiation. J Cutan Pathol 35: 477-483, 2008

12 Morton S, Stevens A and Powell RJ: Basaloid follicular hamartoma, total body hair loss and SLE. Lupus 7: 207-209, 1998.

13 Manfredini M, Arginelli F, Dunsby C, French P, Talbot C, König K, Pellacani G, Ponti G and Seidenari S: High- resolution imaging of basal cell carcinoma: a comparison between multiphoton microscopy with fluorescence lifetime imaging and reflectance confocal microscopy. Skin Res Technol 19: e433443, 2013. 
14 Kimonis VE, Goldstein AM, Pastakia B, Yang ML, Kase R, DiGiovanna JJ, Bale AE and Bale SJ: Clinical manifestations in 105 persons with nevoid basal cell carcinoma syndrome. Am J Med Genet 69: 299-308, 1997.

15 Evans DG and Farndon PA: Nevoid Basal Cell Carcinoma Syndrome. In: GeneReviews ${ }^{\circledR}$ [Internet]. Adam MP, Ardinger HH, Pagon RA, Wallace SE, Bean LJH, Mefford HC, Stephens $\mathrm{K}$, Amemiya A and Ledbetter N (eds.). Seattle, University of Washington, pp. 1993-2017, 2002.

16 Hellani A, Baghdadi H, Dabbour N, Almassri N and Abu-Amero KK: A novel PTCH1 germline mutation distinguishes basal cell carcinoma from basaloid follicular hamartoma: a case report. J Med Case Rep 3: 52, 2009.

17 Howell JB and Caro M: The basal-cell nevus: its relationship to multiple cutaneous cancers and associated anomalies of development. AMA Arch Derm 79: 67-80, 1959.

18 Gartmann H, Groth W and Quinkler C: Multiple basaloid follicular hamartomas in 2 members of a family with GorlinGoltz syndrome. Z Hautkr 64: 915-918, 1989.

19 Ackerman AB: Nevoid basal cell carcinoma syndrome versus generalized basaloid follicular hamartoma syndrome. J Cutan Pathol 36: 603, 2009.

20 Grachtchouk V, Grachtchouk M, Lowe L, Johnson T, Wei L, Wang A, de Sauvage F and Dlugosz AA: The magnitude of hedgehog signaling activity defines skin tumor phenotype. EMBO J 22: 2741- 2751, 2003.

21 Smith KJ and Skelton H: Basaloid follicular hamartomas associated with autoimmune disease: a possible role for retinoids in therapy. J Am Acad Dermatol 49: 1067-1070, 2003.
22 Lo Muzio L, Pastorino L, Levanat S, Musani V, Situm M, Ponti G and, Bianchi Scarra G: Clinical utility gene card for: Gorlin syndrome--update 2013. Eur J Hum Genet 21: 10, 2013.

23 Ponti G, Tomasi A, Pastorino L, Ruini C, Guarneri C, Mandel VD, Seidenari S and Pellacani G: Diagnostic and pathogenetic role of café-au-lait macules in nevoid basal cell carcinoma syndrome. Hered Cancer Clin Pract 10: 15, 2012.

24 Ponti G, Bertazzoni G, Pastorino L, Monari E, Cuoghi A, Bergamini S, Bellei E, Benassi L, Azzoni P, Petrachi T, Magnoni C, Pellacani G, Loschi P, Pollio A, Witkowski AM and Tomasi A: Proteomic analysis of PTCH1+/- fibroblast lysate and conditioned culture media isolated from the skin of healthy subjects and nevoid basal cell carcinoma syndrome patients. BioMed Res Int 2013: 794028, 2013.

25 Ponti G, Pollio A and Pastorino L: Patched homolog 1 gene mutation (p.G1093R) induces nevoid basal cell carcinoma syndrome and non-syndromic keratocystic odontogenic tumors: A case report Oncol Lett 4: 241-244, 2012.

26 da Silva Pierro VS, Marins MR, Borges de Oliveira RC, Cortezzi W, Janini ME and Maia LC: Clinical and oral findings in an Afro-Brazilian family with Gorlin-Goltz syndrome: case series and literature review. Spec Care Dentist 35: 43-50, 2015.

Received October 2, 2017

Revised October 25, 2017

Accepted October 26, 2017 